Document downloaded from:

http://hdl.handle.net/10251/57048

This paper must be cited as:

Sanchez Fandiño, JA.; Muñoz Muñoz, P. (2013). Photonics-based microwave frequency measurement using a double-sideband suppressed-carrier modulation and an InP integrated ring-assisted Mach Zehnder interferometer filter. Optics Letters. 38(21):4316-4319. doi:10.1364/OL.38.004316.

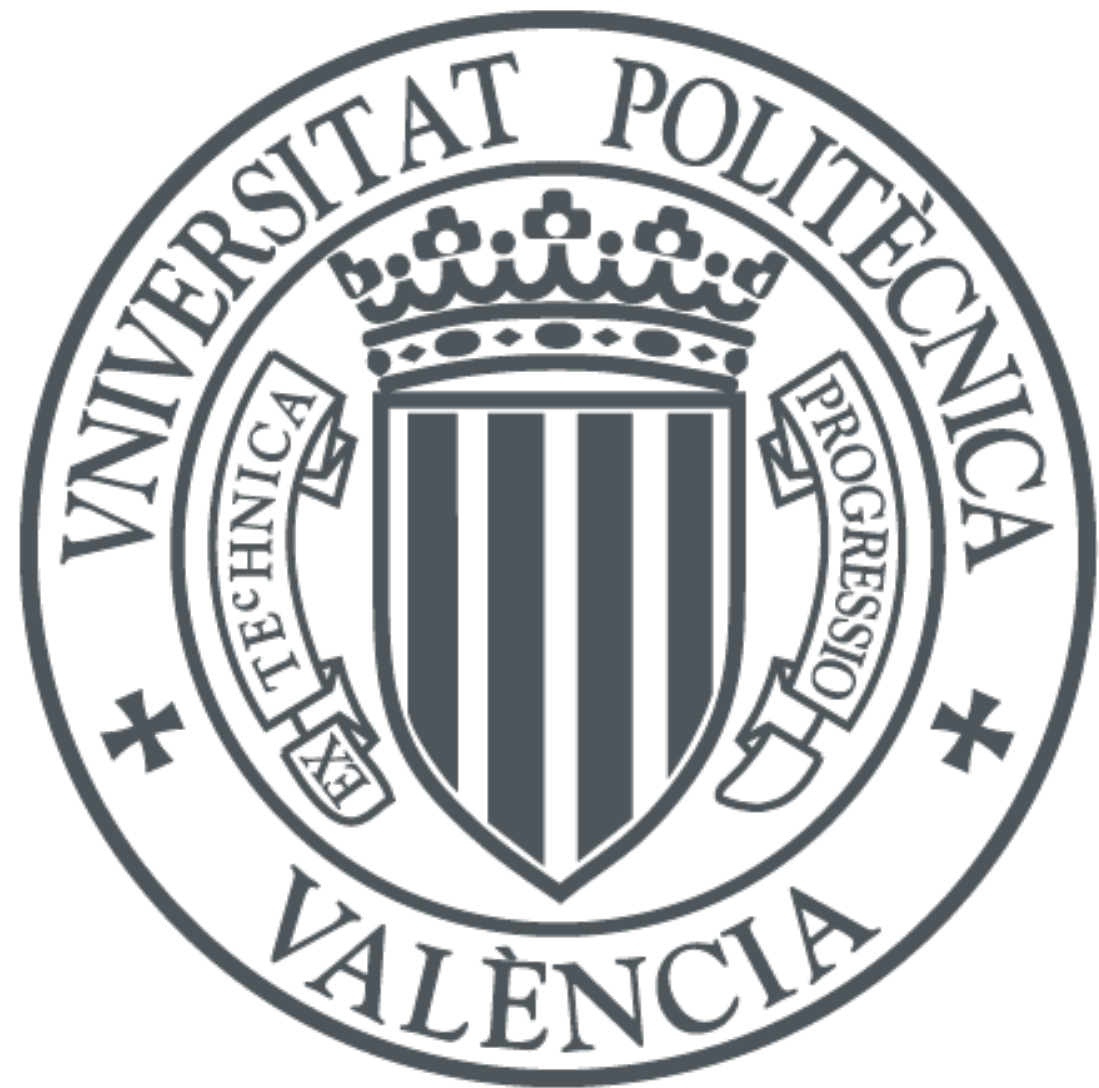

The final publication is available at

http://dx.doi.org/10.1364/OL.38.004316

Copyright Optical Society of America

Additional Information

(C) [2013 Optical Society of America.]. One print or electronic copy may be made for personal use only. Systematic reproduction and distribution, duplication of any material in this paper for a fee or for commercial purposes, or modifications of the content of this paper are prohibited. 


\title{
Photonics-based microwave frequency measurement using a double-sideband suppressed-carrier modulation and an $\ln P$ integrated ring-assisted Mach-Zehnder interferometer filter
}

\author{
Javier S. Fandiño ${ }^{1, *}$ and Pascual Muñoz ${ }^{1,2}$ \\ ${ }^{1}$ ITEAM Research Institute, Universitat Politècnica de València, Camino de Vera s/n, 46022 València, Spain \\ ${ }^{2}$ VLC Photonics S.L., Camino de Vera s/n, 46022 València, Spain
}

compiled: September 25, 2013

\begin{abstract}
A photonic system capable of estimating the unknown frequency of a CW microwave tone is presented. The core of the system is a complementary optical filter monolithically integrated in InP, consisting of a ring-assisted Mach-Zehnder interferometer (RAMZI) with a second-order elliptic response. By simultaneously measuring the different optical powers produced by a double-sideband suppressed-carrier (DSB-SC) modulation at the outputs of the photonic integrated circuit, an amplitude comparison function (ACF) that depends on the input tone frequency is obtained. Using this technique, a frequency measurement range of $10 \mathrm{GHz}(5-15 \mathrm{GHz})$ with a root mean square value of the frequency error lower than $200 \mathrm{MHz}$ is experimentally demonstrated. Moreover, simulations showing the impact of a residual optical carrier on system performance are also provided.
\end{abstract}

OCIS codes: $\quad$ (130.3120) Integrated optics devices; (130.6010) Sensors; (130.7408) Wavelength filtering devices; (060.5625) Radio frequency photonics.

http://dx.doi.org/10.1364/XX.99.099999

Instantaneous frequency measurement subsystems (IFM) are widely used support modules for electronic warfare and electronic intelligence applications. These systems typically provide submicrosecond estimation of the central frequency of a received microwave signal over a broad bandwidth, thus serving as a first step toward characterizing activity in the electromagnetic spectrum and detecting potential threats, such as in RADAR warning equipment [1].

So far, IFM devices have relied on digital and ana$\log$ electronics $[1,2]$. It is for that reason that alloptical approaches have recently attracted much attention, seeking to develop simpler systems with the inherently high bandwidth and electromagnetic immunity of optical technology [3-8]. The basic operation principle behind these photonic techniques consists in establishing a unique relationship between input frequency and the ratio of two measured powers. This mapping between frequency and power (which can be either optical or $\mathrm{RF}$ ), is commonly referred to in the literatue as amplitude comparison function (ACF) [3]. Since detection of $\mathrm{RF}$ power relies on expensive electronic devices, such as digital logarithmic video amplifiers, optical power-based approaches stand out as a promising alternative that only require the use of low-speed photodiodes and simple electronics. Several of these all-optical approaches

* Corresponding author: jasanfan@upvnet.upv.es have been experimentally demonstrated so far, including IFM systems using photonic Hilbert transforms [4] and complementary filter pairs [5, 6]. Moreover, some photonic integrated circuits (PICs) for IFM have also been recently reported $[3,7]$, which can be considered as part of the emerging field of integrated microwave photonics [9].

In this work, a PIC-based system for estimating the frequency of a CW microwave signal is experimentally demonstrated. Exploiting the complementary responses of a ring-assisted Mach-Zehnder interferometer (RAMZI) filter monolithically integrated in InP, a nonambiguous frequency measurement range of $10 \mathrm{GHz}(5-$ $15 \mathrm{GHz}$ ) is achieved. Besides, an experimental root mean square value (RMS) of the frequency error lower than $200 \mathrm{MHz}$ is demonstrated over the same bandwidth. Additionally, simulations showing the negative impact of a residual optical carrier on system performance are provided. Thanks to the great flexibility in both active and passive integration boasted by $\mathrm{InP}$ technology, this approach holds great promise for enabling future photonics-based IFM systems with lower cost and complexity over current electronic solutions.

The operation principle behind our IFM system was first proposed and demonstrated by Zou et al. [5]. First, an optical carrier is introduced into a low-biased MachZehnder modulator (MZM) driven by a CW microwave signal with unknown frequency. Since the optical carrier is greatly suppressed because of the low bias, a double- 


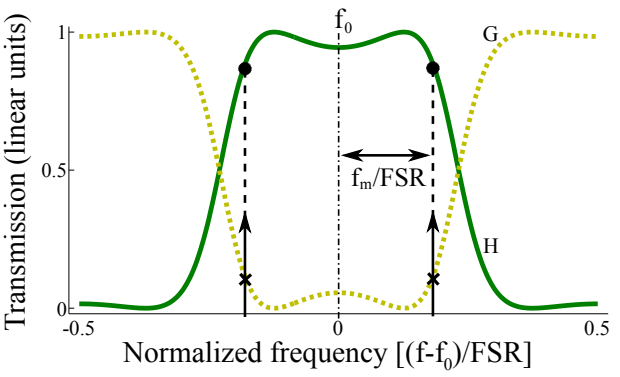

(a)

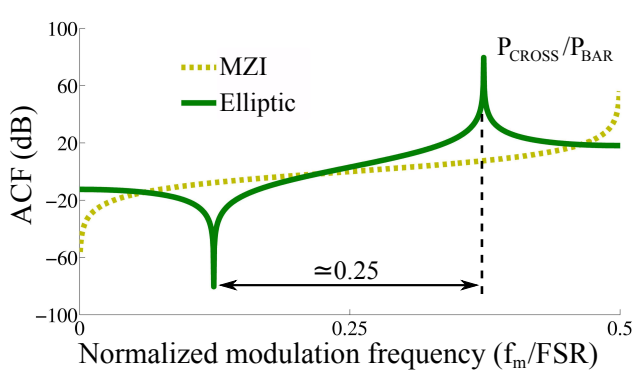

(b)

Figure 1. (a) Operation principle of the IFM system. Here, H (solid line) represents the power transmission response (linear units) of the elliptic filter (bar), while G (dashed line) represents its corresponding complementary response (cross). (b) Simulated ACFs for both the elliptic filter (solid line) and a MZI (dashed line).

sideband suppressed-carrier (DSB-SC) modulation with two sidebands that are $\pm f_{m}$ away from the optical carrier $\left(f_{0}\right)$ is generated. The signal is then introduced into a complementary optical filter. This is, a filter where the sum of the optical powers present at its two outputs remains constant, such as in a MZI. We shall call the filter outputs as bar and cross. Now, since the sidebands at each output experience different attenuations (see Fig. 1- $a$ ), two distinct optical powers are photodetected. Assuming perfect optical carrier suppression, it can be indeed shown that their ratio is independent of both laser and input RF power [5]. It only depends on the filter response and the modulation frequency of the input signal, which is precisely the parameter of interest. The resulting ACF obtained by using this technique can be expressed in its most general form as:

$A C F\left(f_{m}\right)=\frac{P_{\text {cross }}\left(f_{m}\right)}{P_{\text {bar }}\left(f_{m}\right)}=\frac{\left|G\left(f_{0}+f_{m}\right)\right|^{2}+\left|G\left(f_{0}-f_{m}\right)\right|^{2}}{\left|H\left(f_{0}+f_{m}\right)\right|^{2}+\left|H\left(f_{0}-f_{m}\right)\right|^{2}}$

where $f_{m}$ is the microwave input frequency to be estimated, $f_{0}$ is the frequency of the optical carrier, and $H(f) / G(f)$ symbolize the complementary transfer functions of the filter (bar and cross, respectively).

While the previously reported approaches based on complementary filters have relied on MZI-like structures [5, 6], our RAMZI filter (Fig. 2- $a$ ) has a second-order elliptic response. It is composed of a Mach-Zehnder

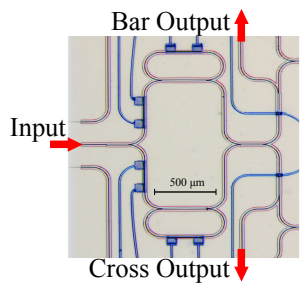

(a)

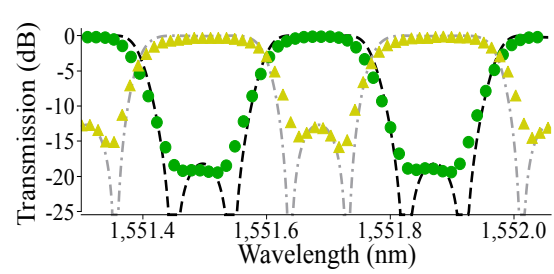

(b)
Figure 2. (a) Filter photomicrograph. (b) Normalized transfer functions measured for both bar (circles) and cross responses (triangles). Simulation results are also shown (dashed and dotted-dashed lines, respectively). interferometer loaded with one ring on each arm [10]. Because of its higher roll-off (faster transition between the passband and the stopband), the elliptic filter results into a steeper ACF as compared with a single-stage MZI (see Fig. 1-b). As pointed out in [8], this implies that a higher frequency resolution can be achieved for the same free spectral range (FSR), since ACF slope and frequency resolution are directly related. However, this comes at the expense of a reduction in the maximum monotonic bandwidth of the ACF. In this case, it is reduced from $50 \%$ of the FSR when using a MZI to nearly $25 \%$. It must be noted that monotonicity and power independence of the ACF are fundamental conditions for unambiguously determining input frequency, since they allow to establish a unique mapping between the parameter of interest $\left(f_{m}\right)$ and a measured power ratio.

The experimental procedure can be described as follows. First, the filter transfer function is measured and adjusted to match as closely as possible its ideal simulated response. This is done by coupling an ASE light source into the chip while recording the filter transmission spectrum at the output with a high resolution Optical Spectrum Analyzer (OSA), and then normalizing it with respect to a reference waveguide present on the same chip. During this process, the filter response is manually adjusted by means of on-chip thermo-optic heaters, which allow for compensation of the optical phase errors that result from the non-uniformities of the fabrication process. After adjustment, the currents injected into the heaters are recorded and kept fixed, while the chip is maintained at a constant temperature $\left(\simeq \pm 0.1^{\circ} \mathrm{C}\right)$ with a closed-loop temperature controller (Newport, LDT-5412) using a $10 \mathrm{k} \Omega$ NTC thermistor and a Peltier cell. The total estimated electrical power employed for chip tuning is $163.3 \mathrm{~mW}$. Measured spectra after optimization are shown in Fig. 2- $b$, normalized with respect to the filter insertion losses. Its FSR is $0.375 \mathrm{~nm}(\simeq 46.7 \mathrm{GHz} @ 1550 \mathrm{~nm})$, which implies a monotonic measurement range $(\simeq 25 \%$ of the FSR) of about $11.68 \mathrm{GHz}$. Filter insertion losses, as measured with respect to a reference waveguide located on the same chip, are around $6.5 \mathrm{~dB}[10]$. Total insertion losses 


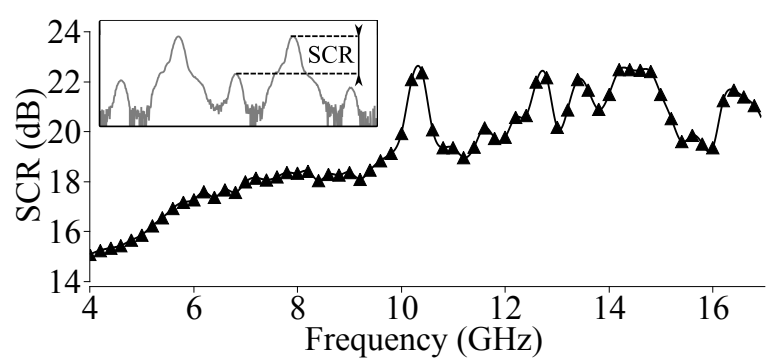

Figure 3. Measured Sideband-to-Carrier ratio versus modulation frequency.

of the chip (input to output) are higher than $20 \mathrm{~dB}$.

Once the filter has been optimized, the wavelength of a CW tunable laser (Yenista Tunics T100R, $15 \mathrm{~mW}$ ) is first set to match the center wavelength of the filter $(1551.684 \mathrm{~nm})$. The optical source is then introduced into an external MZM (Sumitomo DEH1.5-40PD-ADC, $\mathrm{V}_{\pi}=5.5 \mathrm{~V}, 30 \mathrm{GHz}$ bandwidth) driven by a CW microwave signal $(+7 \mathrm{dBm}$, Agilent PNA E8364A) and manually low-biased with a voltage source. The generated DSB-SC modulation is subsequently polarization filtered (TE mode) by a free space polarizer, and lenscoupled into the chip. After passing through the on-chip filter, the optical power is collected at the output with a lensed fiber (Yenista Optics) and directed into an optical power meter. Finally, the data is stored for further post-processing. Multiple data points are collected by conveniently automating the setup with a General Purpose Interface Bus (GPIB), so as to estimate temporal stability and frequency error uncertainty. A total of 20 frequency sweeps (from 0.2 to $20 \mathrm{GHz}$, in steps of $0.2 \mathrm{GHz}$ ) are performed over a $20 \mathrm{~min}$ interval for each individual output port (40 min in total).

The raw data is post-processed and 20 different ACF curves are obtained, one for each frequency sweep. Their sample mean is then calculated in order to get the best possible estimate of the system ACF. The result is plotted in Fig. 4- $a$ (solid line). As predicted by simulations, a measurement range of about $11 \mathrm{GHz}(5-16 \mathrm{GHz})$ is obtained. Not only that, the peaks and dips of the ideal ACF shown in Fig. 1- $b$ have been considerable reduced. In order to understand why this happens, note that the ACF shown in Fig. 1- $b$ was obtained by simulating the behaviour of the setup under ideal circumstances. It was assumed that the optical carrier is completely suppressed and that the filter responses are perfectly implemented and have no losses. However, this is not the case in practice. First of all, the measured transfer functions of the filters do not present the same deep notches in the stopband. Second, there always remains a certain amount of optical carrier power after the low-biased MZM, which depends on the extinction ratio of the device. In fact, the presence of this residual power directly affects the shape of the ACF. The higher the optical carrier power relative to the power of the sidebands (mea- sured as the Sideband-to-Carrier ratio, or SCR), the flatter and smoother the measured ACF is, since the power ratio tends to be more constant.

In order to account for this non-ideal behaviour, the SCR was measured during the experiments by directly connecting the output of the modulator to the OSA (Fig. 3). Now, by combining both the non-infinite SCR and the real responses of the filters, a more realistic simulation can be run. Simulations for different input RF powers, a parameter which was not varied during the experiment, can also be obtained. The results are plotted in Fig. 4- $a$, which clearly show a better agreement with the measured data and illustrate how the ACF gets flatter as the RF power is decreased.

Note that, due to the RF power dependence of the $\mathrm{ACF}$, a unique calibration curve from where input frequencies can be inferred given a measured power ratio does not exit. If one of them is chosen as such, microwave signals with equal frequency but RF powers that differ from that used for calibration will result into a biased frequency estimate. Besides, any spurious power fluctuations (such as those caused by mechanical or thermal drifts), will lead to a spread in the measurement, typically characterized by a standard deviation. Both effects thus need to be taken into account when estimating the frequency error of the system. As the RMS value of the frequency error takes into account both effects (bias and standard deviation), it serves as a good figure of merit that gives an idea of the error statistics during the measurement process.

The RMS of the frequency error can be computed as follows. First, a fitting of the measured mean ACF $(+7 \mathrm{dBm}$, solid line in Fig. 4- $a)$ is chosen as the calibration curve for frequency estimation. As explained before, for every input modulation frequency used to test the system, 20 different power ratios were measured. Thus, 20 different frequencies can be estimated by looking up these ratios in the fitted curve. The difference between the resulting value and the one supplied by the Microwave Network Analyzer gives us the frequency error. Now, the RMS error can be computed and plotted against input frequency (triangles in Fig. 4- $b$ ).

In order to extend this procedure for different RF powers, where no data is available, we must further assume that the fluctuations in optical power are similar as those measured during the experiment. By combining these with the simulated ACFs of Fig. 4- $a$, an estimation of the RMS value for different input RF powers is obtained. In this case, the calibration curve chosen as a reference is the simulated ACF for an input RF power of $+7 \mathrm{dBm}$. Final results are shown in Fig. 4- $b$. The measured RMS error remains lower than $200 \mathrm{MHz}$ for a bandwidth of about $10 \mathrm{GHz}(5-15 \mathrm{GHz})$. This is mainly attributed to the effect of the different noise processes affecting the measured optical power. Also note that the frequency error rises around 5 and $16 \mathrm{GHz}$ due to a reduction in the ACF slope. As regards the simulation data, variations of input RF power lead to a significant increase in 


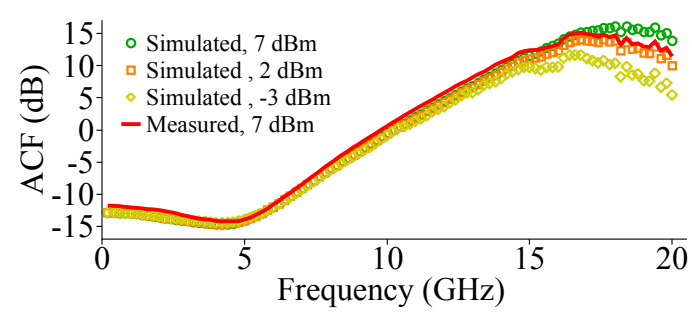

(a)

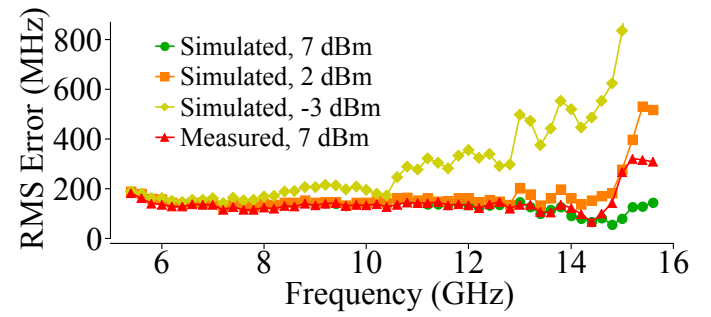

(b)

Figure 4. (a) Measured mean ACF (solid line) and simulated mean ACFs for different input RF powers. (b) RMS value of the frequency error as a function of modulation frequency.

error. At lower frequencies the simulated ACFs are similar to the reference curve. Thus, the bias error is low and the RMS error is mainly given by power noise. At higher frequencies, the ACFs tend to diverge so the bias contribution to the RMS error increases. In fact, an estimated RMS error above $600 \mathrm{MHz}$ is obtained over the same bandwidth for a RF power of $-3 \mathrm{dBm}$, which is just $10 \mathrm{~dB}$ lower as that used in the experiment.

The measured frequency error in our experiment is similar to previously reported approaches based on integrated optics [3, 7], with 93.6 MHz and $200 \mathrm{MHz}$, respectively. As an advantage, it only requires the use of low-speed photodiodes and simple electronics, as compared with [3] where RF power detection is employed. Moreover, operation is simpler and faster than that presented in [7], since there is no need to sweep the chip temperature. On the other side, improvements in carrier suppression and reduction of random power fluctuations must be achieved in order to ensure a low frequency error. Thanks to recent advances in photonic integration, these impairments might well be solved in the near future, both by fitting all active and passive components on a single InP chip (reducing external noise sources) and applying advanced techniques for carrier suppression in integrated MZMs. In fact, suppression ratios as high as $39 \mathrm{~dB}$ have been recently reported [11].

A photonics-based system that is capable of estimating the unknown frequency of a $\mathrm{CW}$ microwave signal has been reported. Its operation principle relies on exploiting the different attenuations that a dual-sideband suppressed-carrier optical modulation experiences when passing through a RAMZI filter integrated in InP. Using this approach, a frequency measurement bandwidth of $10 \mathrm{GHz}(5-15 \mathrm{GHz})$ has been experimentally demonstrated, achieving a root mean square value of the frequency error lower than $200 \mathrm{MHz}$. Simulations have also been performed that demonstrate the negative influence of a reduced optical Sideband-to-Carrier ratio in system performance, with a RMS error above $600 \mathrm{MHz}$ when the RF power is reduced by $10 \mathrm{~dB}$. Similar to other op- tical power-based approaches, this system only requires low-speed photodetectors and electronics, and it could be easily integrated on a single InP chip. With further advances in carrier suppression techniques, InP technology presents a promising opportunity for the realization of future high bandwidth, all-optical and reduced footprint IFM systems.

This work was supported by projects TEC2010-21337 (ATOMIC), FEDER UPVOV10-3E-492 and UPVOV083E-008. J.S. Fandiño was supported by Grant FPU2010 (ref: AP2010-1595). The authors acknowledge the use of Phoenix Software tools for design of the InP PIC, which was manufactured within the EuroPIC FP7 project (ref: NMP 228839-2).

\section{References}

[1] P. L. Herselman and J. E. Cilliers, S. Afr. J. Sci. 102, 345 (2007).

[2] G.-C. Liang, C.-F. Shih, R. Withers, B. Cole, and M. Johansson, IEEE Trans. Microw. Theory Tech. 44, 12891299 (1996).

[3] D. Marpaung, IEEE Photon. Technol. Lett. 25, 837-840 (2013).

[4] H. Emami, N. Sarkhosh, L. A. Bui, and A. Mitchell, Opt. Express 16, 13707-13712 (2008).

[5] X. Zou, H. Chi, and J. Yao, IEEE Trans. Microw. Theory Tech. 57, 505-511 (2009).

[6] B. Lu, W. Pan, X. Zou, B. Luo, L. Yan, X. Liu, and S. Xiang, IEEE Photon. Technol. Lett. 25, 500-503 (2013).

[7] H. Guo, G. Xiao, N. Mrad, and J. Yao, IEEE Photon. Technol. Lett. 21, 45-47 (2009).

[8] X. Zou and J. Yao, IEEE Photon. Technol. Lett. 20, 1989-1991 (2008).

[9] D. Marpaung, C. Roeloffzen, R. Heideman, A. Leinse, S. Sales, and J. Capmany, Laser \& Photonics Reviews 7, 506-538 (2013).

[10] J. S. Fandino, J. D. Domenech, P. Munoz, and J. Capmany, Opt. Express 21, 3726-3736 (2013).

[11] Y. Ogiso, Y. Tsuchiya, S. Shinada, S. Nakajima, T. Kawanishi, and H. Nakajima, IEEE Photon. Technol. Lett. 22, 941-943 (2010). 